\title{
Investigating the Effect of Inclination Angle of Magnetic Field Vector on Silicon PV Modules
}

\author{
Dioari Ulrich Combari ${ }^{D},{ }^{1,2}$ Emmanuel Wendsongré Ramde, ${ }^{3}$ Bruno Korgo, ${ }^{1}$ \\ Ramatou Saré, ${ }^{1}$ Martial Zoungrana $\oplus^{\circledR}{ }^{1}$ and Issa Zerbo $\oplus^{1}$ \\ ${ }^{1}$ Laboratory of Thermal and Renewable Energy, Department of Physics, Unit of Training and Research in Pure and Applied Sciences, \\ University Joseph Ki-Zerbo, Ouagadougou, Burkina Faso \\ ${ }^{2}$ Polytechnic University of Ouagadougou, Ouagadougou, Burkina Faso \\ ${ }^{3}$ Department of Mechanical Engineering and The Brew-Hammond Energy Centre, Kwame Nkrumah University of Science \\ and Technology, Kumasi, Ghana
}

Correspondence should be addressed to Dioari Ulrich Combari; dioariulich@gmail.com

Received 2 September 2020; Revised 4 March 2021; Accepted 25 March 2021; Published 8 April 2021

Academic Editor: Jinn Kong Sheu

Copyright (c) 2021 Dioari Ulrich Combari et al. This is an open access article distributed under the Creative Commons Attribution License, which permits unrestricted use, distribution, and reproduction in any medium, provided the original work is properly cited.

\begin{abstract}
In earlier studies, we have shown theoretically and experimentally that magnetic fields (MFs) have negative impact on silicon PV module (photovoltaic module). A noticeable decline in photocurrent with a slight increase in photovoltage was observed. Also, how those fields affected other key module's parameters was also studied. These studies concluded that an increase in the magnitude of the MF resulted in the decrease of the efficiency of the silicon PV module. The previous experimental studies assumed that the MF vector formed zero angle of inclination with respect to the photosensitive face of the module. They did not factor in any effect that could be observed when the field vector is inclined. The present experimental work is an attempt to fill that gap. The characteristic curves of the PV module were plotted in the same system of axis for different values of the inclination angle of the MF vector. Correspondingly, the characteristic values $\left(P_{\max }, I_{\max }, V_{\max }, I_{\mathrm{sc}}\right.$, and $\left.V_{\mathrm{oc}}\right)$ of the PV module were also determined. These parameters then allowed the calculation of the efficiency of the module, its fill factor, and the equivalent circuit series and shunt resistances. It is observed that the module efficiency increases with the inclination of the MF vector, indicating that the effect of the MF on the PV module is reduced when its vector aligns towards a direction that is perpendicular to the base of the module. For example, when $\alpha$ moves from 0 to $90^{\circ}$, the power output and consequently the efficiency of the PV module relatively increase of $14 \%$.
\end{abstract}

\section{Introduction}

In reality, PV modules are almost never operated under standard test conditions (module temperature of $25^{\circ} \mathrm{C}$, a solar Irradiance of 1000 watt per $\mathrm{m}^{2}$ and an air mass of 1.5). Their expected performance therefore is also almost never achieved. This must be added to the problems of degradation caused by their exposure to ultraviolet radiation, high temperature gradients [1,2], dust and humidity [3], etc. Furthermore, it is theoretically established that magnetic fields influence negatively the performance of PV cells and modules [4-8].
In order to support the theoretical studies, some researchers have experimentally investigated the influence that magnetism has on the behavior of PV cells (photovoltaic cells) or PV modules. Betser et al. [9] conducted an experiment that led to the establishment of some particularly interesting equations in magnetotransport. Their study resulted, among other things, in the determination of the impact that external magnetic fields create on the current that passes through the base of a heterojunction bipolar transistor.

The experimental studies of Erel $[10,11]$ have shown that magnetic fields influence the electrical parameters of any of the three main types of PV cells, namely, monocrystalline, 
polycrystalline, and amorphous. The influence was more pronounced on the open-circuit voltage $V_{\mathrm{oc}}$ and on the short-circuit current $I_{\mathrm{sc}}$. Indeed, he arrived at the conclusion that when these three types of PV cells are illuminated with a tungsten lamp of variable intensity, $V_{\mathrm{oc}}$ and $I_{\mathrm{sc}}$ decrease against the intensity of the MF applied to the PV cell [10].

In another study, Vardanyan et al. [12] developed a method for evaluating the diffusion length, the diffusion coefficient, the mobility of charge carriers, and the velocity of the back-surface recombination of a bifacial PV cell. Its base was positively doped, so as to obtain an n-p type photocell. Illuminated with a monochromatic light, its recombination parameters were obtained by gauging the short-circuit photocurrent at two separate wavelengths, first in the presence and then in the absence of a magnetic field, and using the expression of electron photocurrent and its associated parameters.

Combari et al. [13] performed some experimental studies with the aim of assessing the effect of the intensity of an external MF on the current-voltage and power-voltage response of a monocrystalline silicon solar module subjected to a constant solar irradiation. They found that the efficiency of the module drops as the intensity of the MF goes up. This was as a result of a considerable drop in all currents that the PV module delivers even though there was a slight increase in voltages.

Fathabadi [14] has conducted theoretical and experimental studies of the effect of electromagnetic field produced by high-tension transmission lines on the yield of solar modules placed in their vicinity. He found that the effect of the electrical component of the electromagnetic wave that these lines induce is nil while the magnetic component reduces the power output generated by the PV module. In another theoretical and experimental studies [15], he examined the effect of the electric and magnetic fields of an alternating current on the electrical production of a PV cell. He found that this electric field has no effect on their open-circuit voltage and electric power. However, these two parameters decrease with the alternating magnetic field. He also conducted a comparative study of the effect of the MF on the photocurrent of three types of PV cells (organic, dye-sensitized, and silicon solar cells) [16]. He found that the density of the photocurrent of the organic heterojunction cell increases with the increase of the MF whereas its impact on that of the dye-sensitized and silicon solar cells causes a decrease.

In their 3D study, Sourabié et al. [17] showed theoretically that the inclination $\theta$ of a magnetic field vector has an effect on the output of polycrystalline silicon PV cell. Their study showed that the output of the polycrystalline silicon cell increases when $\theta$ moves from 0 to $90^{\circ}$.

In this paper, we experimentally tested the effect of the inclination angle of a MF vector on the performance of a monocrystalline silicon PV module. The intensity $B$ of the MF was held constant while measurements were being taken for different inclination angles $\alpha$ of the MF vector. The current-voltage and power-voltage characteristic curves were plotted and then analyzed in order to measure the degree of impact of the inclination angle of the MF vector on the operation of the PV module. The characteristic parameters
$\left(P_{\max }, I_{\max }, V_{\max }, I_{\mathrm{sc}}\right.$, and $\left.V_{\mathrm{oc}}\right)$ of the PV module and then the fill factor, the power conversion efficiency, the series, and shunt resistances were worked out.

\section{Materials and Methods}

Throughout the experiment, a constant solar irradiation was kept incident upon the PV module while it was subjected to a MF of constant intensity. Though the intensity of the MF was held constant, its vector $\vec{B}$ was free to rotate on the $x y$ plane while forming an angle $\alpha$ with the $y$ axis.

The PV module used in this study consisted of 36 cells mounted in series. It was assumed that each cell was subjected to the magnetic field $\vec{B}$ inclined at an angle $\alpha$ with the plane parallel to its photosensitive area as depicted in Figure 1. $\alpha$ was made to vary as $\vec{B}$ sweeps planes that are parallel to the $x y$ plane. The divergence of $\vec{B}$ within the PV cell was assumed to be zero.

PV modules are generally made up of $\mathrm{n}$ sets of $\mathrm{Np}$ parallel strings mounted in series, with each string having Ns number of cells. The PV module considered in this study is made up of 36 cells all mounted in series. In this case, $n=\mathrm{Np}=1$ and $\mathrm{Ns}=36$.

Each cell has a surface $S$ of $7.5 \mathrm{~cm}^{2}$. The synoptic diagram of this PV module is given in Figure 2 [8].

From V1 to V36: voltage across each of the 36 cells of the PV module.

The layout of the experimental set-up used in this study is illustrated in Figure 3 [13].

Unlike our previous experiment where the angle of inclination $\alpha$ of the MF vector was kept zero throughout, in the present work, $\alpha$ is made to vary and the current-voltage $(\mathrm{I}-\mathrm{V})$ as well as the power-voltage $(\mathrm{P}-\mathrm{V})$ characteristics are determined.

\section{Results and Discussion}

3.1. Current-Voltage Characteristic Curves of the PV Module against Angle of Inclination $\alpha$. With the experimental set-up described in the previous section, the current of the PV module was measured against its voltage for five different values of the inclination angle $\alpha$ of the MF vector. Intensities of the solar irradiance and of the magnetic field were kept constant at $630 \mathrm{~W} \cdot \mathrm{m}^{-2}$ and $15 \mathrm{mT}$, respectively, and the average ambient temperature was about $39^{\circ} \mathrm{C}$. Figure 4 represents the current-voltage (I-V) curves for each value of the inclination angle $\alpha$ of the MF vector.

As it can be observed in Figure 4, the intensity of the current increases with the inclination $\alpha$ of the MF vector. Indeed, the magnetic field vector has a horizontal component $B_{y}$ and a vertical component $B_{x}$. Component $B_{y}$ is parallel to the junction of each cell as depicted in Figure 1. It is the component that acts on the minority carriers (electrons) through the Lorentz force, thus negatively affecting the performance of the PV module. As its value decreases with increasing angle of inclination $\alpha$, the deviation of the electrons towards the lateral surfaces decreases, resulting in a decrease of the 


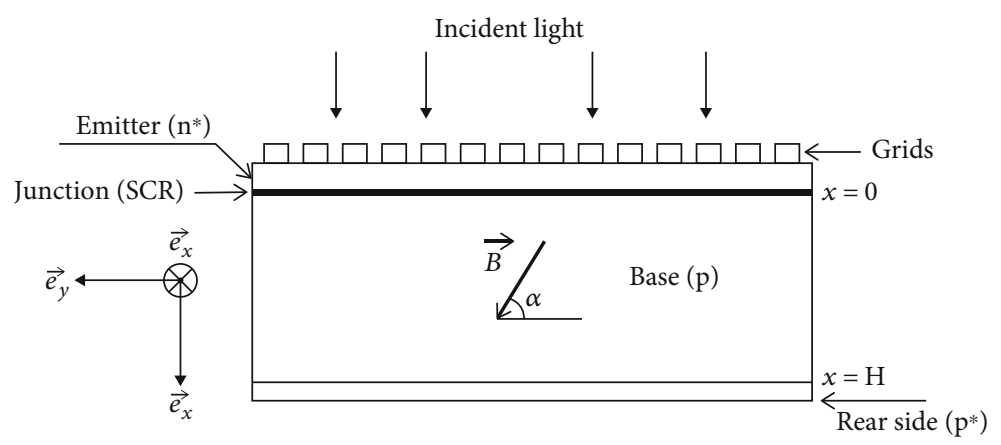

Figure 1: PV cell subjected to a constant multispectral illumination and under the effect of the inclination $\alpha$ of the applied MF vector.

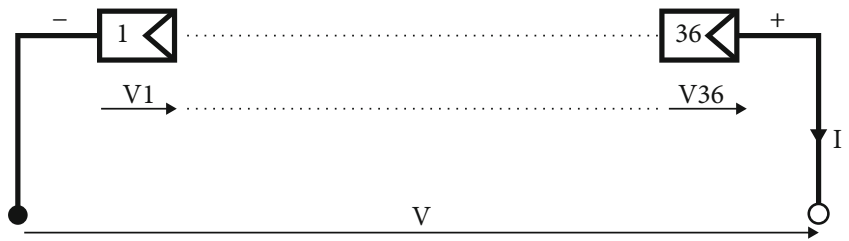

Figure 2: Synoptic diagram of the PV module.

accumulation of electrons near the junction. The net effect is an increase of the photocurrent of the PV module. Likewise, as the value of component $B_{y}$ decreases with increasing $\alpha$, it results in the drop of the concentration gradient of the minority carriers between the front and the rear faces of the cells. Consequently, more photogenerated charges can cross the junction of the cells and participate in the photocurrent. A slight decrease in voltage is then observed.

In summary, the increase of the inclination angle $\alpha$ of the MF vector results in an increase of the intensity of the current in general and of the short-circuit current, in particular. In contrast, the open-circuit voltage decreases. Overall, the net influence of the MF on the operation of the PV module decreases with the increase of the inclination angle $\alpha$ of its vector.

3.2. Power-Voltage Characteristic Curves against Angle of Inclination $\alpha$. Similarly, the power provided to the load by the PV module was also measured against its voltage for five different values of the inclination angle $\alpha$ of the MF vector while the intensities of the solar irradiance and of the magnetic field were kept constant, as set before. The results are represented in Figure 5.

As it can be seen in Figure 5, the power-voltage characteristics show that the power output of the PV module under the influence of the MF increases with the inclination angle $\alpha$ of the field vector. This is in line with the results presented in the previous section. Indeed, the effect of the MF becomes low as its field vector tilts more with respect to the photosensitive face of the PV module. Indeed, when the field vector becomes perpendicular to the photosensitive face (case of $\alpha$ $=90^{\circ}$ ), the trend of the power-voltage curve is almost the same as that obtained with no magnetic field; that means that the effect of the MF on the PV module ceases completely. In contrast, when the field vector aligns with the photosensitive face (case of $\alpha=0^{\circ}$ ), the influence of the MF becomes maxi- mum; hence, the power output of the module reaches its lowest value.

3.3. Electrical Performance of the PV Module against Angle of Inclination $\alpha$. The superposition of the current-voltage (I-V) and the power-voltage $(\mathrm{P}-\mathrm{V})$ curves of the photovoltaic module on the same system of axis allows the determination of the characteristic parameters $\left(P_{\max }, I_{\max }, V_{\max }, I_{\mathrm{sc}}\right.$, and $\left.V_{\mathrm{oc}}\right)$ of the PV module. Once they are known, they are in turn used to calculate the fill factor FF and the efficiency $\eta$ of the PV module [8].

Figure 6 confirms the results illustrated in Figures 4 and 5. The figure displays the voltage, the current, and power output of the PV module at the maximum power point as well as the open-circuit voltage and the short-circuit current for 5 different values of the angle of inclination of the MF vector. As the angle of inclination of the MF vector increases, the voltage decreases marginally while the current increases significantly. The net effect is an increase of power output which reaches a maximum at $\alpha=90^{\circ}$. The maximum power output relatively increases of $3 \%, 7 \%, 10 \%$, and $14 \%$ when $\alpha$ increases from 0 to $30^{\circ}, 0$ to $45^{\circ}, 0$ to $60^{\circ}$, and 0 to $90^{\circ}$, respectively.

Since the PV module was subjected to an average solar irradiance of $630 \mathrm{~W} \cdot \mathrm{m}^{-2}$ and the surface of the PV module $S$ was $0.027 \mathrm{~m}^{-2}$, then the incident solar irradiance is $P_{i}=630 \times 0.027$, i.e., 17 watt. This is useful in the calculation of the efficiency which is given by Equation (1).

$$
\eta(\alpha)=\frac{P_{\max }(\alpha)}{P_{i}}
$$

The fill factor is computed with Equation (2).

$$
\mathrm{FF}(\alpha)=\frac{V_{\max }(\alpha) \cdot I_{\max }(\alpha)}{V_{\mathrm{oc}}(\alpha) \cdot I_{\mathrm{sc}}(\alpha)}=\frac{P_{\max }(\alpha)}{V_{\mathrm{oc}}(\alpha) \cdot I_{\mathrm{sc}}(\alpha)}
$$

The clustered column chart of Figure 7 presents the fill factor and the efficiency of the PV module for different angles of inclination of the MF vector.

The values of the fill factor FF decrease with the increase of the inclination angle $\alpha$ of the MF vector as it can be seen from Figure 7 . The fill factor FF drops slightly down with increasing $\alpha$. As the MF vector tilts from 0 to $30^{\circ}, 0$ to $45^{\circ}$, 0 to $60^{\circ}$, and 0 to $90^{\circ}, \Delta \mathrm{FF} / \mathrm{FF}$ regresses of $3.5 \%, 4.1 \%, 5.1 \%$, 


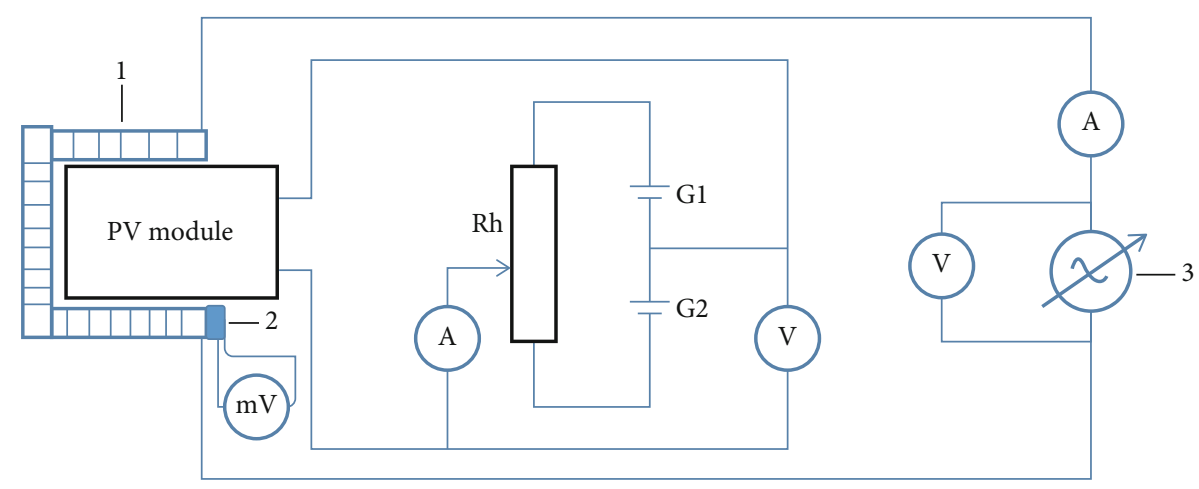

Figure 3: Layout of the experimental set-up. 1: inductance; 2: probe; 3: autotransformer.

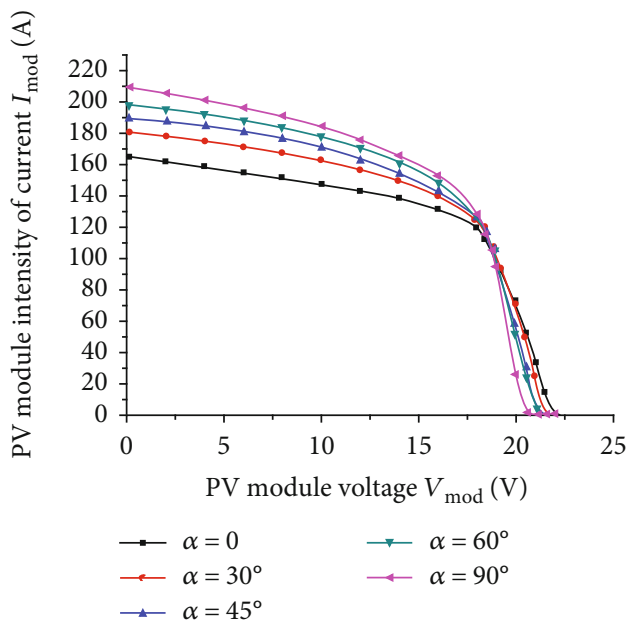

FIGURE 4: Current-voltage characteristic curves vs. inclination angle $\alpha ; E=630 \mathrm{~W} . \mathrm{m}^{-2}$ and $B=15 \mathrm{mT}$.

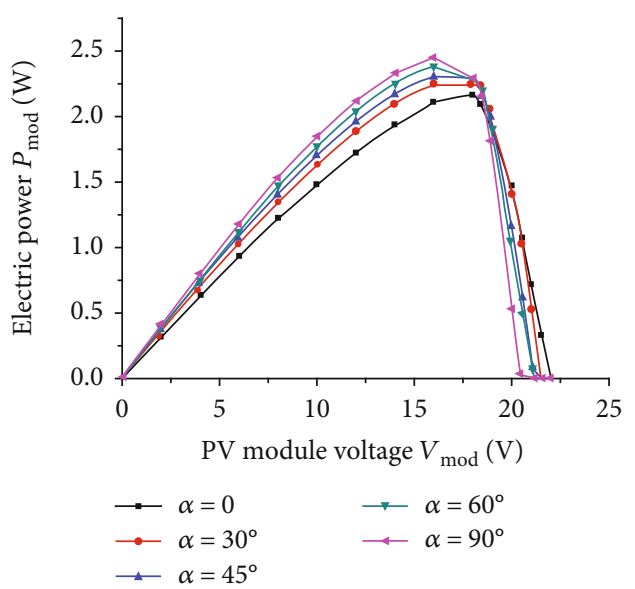

Figure 5: Power-voltage characteristic curves of the PV module vs. inclination angle $\alpha ; E=630 \mathrm{~W} . \mathrm{m}^{-2}$ and $B=15 \mathrm{mT}$.

and $5.4 \%$, respectively. Indeed, as stated before, as the angle increases, the short-circuit current increases at a rate higher than the rate at which the open-circuit voltage decreases. This results in a drop of FF as it is mathematically anticipated from Equation (2).
Conversely still in Figure 7, the efficiency increases in the same order of magnitude as the increase in power output of the PV module, that is, 3\%, 7\%, 10\%, and $14 \%$, when $\alpha$ increases from 0 to $30^{\circ}, 0$ to $45^{\circ}, 0$ to $60^{\circ}$, and 0 to $90^{\circ}$, respectively.

3.4. Series and Shunt Resistances against the Inclination Angle $\alpha$. The series and shunt resistances were computed by adapting the equations recommended by Kareem et al. [18] and by using the characteristic values earlier provided.

$$
\begin{aligned}
& \mathrm{R}_{\text {shmod }}(\alpha)=\frac{\mathrm{V}_{\max }(\alpha)}{\mathrm{I}_{s c}(\alpha)-\mathrm{I}_{\max }(\alpha)} . \\
& \mathrm{R}_{\text {smod }}(\alpha)=\frac{\mathrm{V}_{o c}(\alpha)}{\mathrm{I}_{s c}(\alpha)}-\frac{\mathrm{V}_{\max }(\alpha)}{\mathrm{I}_{\max }(\alpha)} .
\end{aligned}
$$

The calculated values of the two resistances of the equivalent circuit for 5 different values of the angle of inclination $\alpha$ of the MF vector are depicted in Figure 8.

As it can be observed from Figure 8, the more the angle of inclination $\alpha$ widens, the more the series resistance and the shunt resistance decrease. This means that the greater the angle of inclination $\alpha$, the lesser the influence of the MF on both resistances. As an illustration, when $\alpha$ moves from 0 to $90^{\circ}$, the relative decrease of the series and shunt resistances are $47 \%$ and $36 \%$, respectively. In fact, when the MF vector becomes normal to the photosensitive face of the module (case of $\alpha=90^{\circ}$ ), the series and shunt resistances assume almost the same values as those recorded in the absence of magnetic field.

An increase of the short-circuit current means a reduction of the series resistance of the PV module. On the other hand, a decrease of the open-circuit voltage means a decrease in the shunt resistance which prevents the electrons from crossing the junction (leakage current), leading to a lesser storage of carriers at the junction. The simultaneous decrease of the series and shunt resistances of the PV module, when $\alpha$ changes from 0 to $90^{\circ}$, confirms the gradual drop of the magnetoresistance which eventually disappears at $\alpha=90^{\circ}$. 


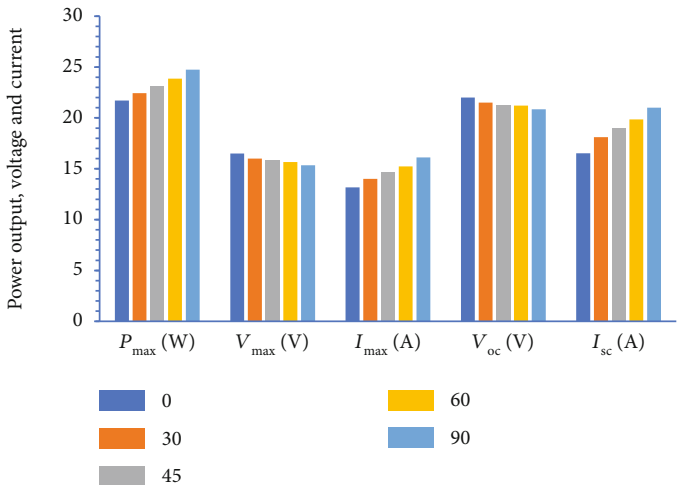

Figure 6: Characteristic parameters $\left(P_{\max }, I_{\max }, V_{\max }, I_{\mathrm{sc}}\right.$, and $\left.V_{\mathrm{oc}}\right)$ of the PV module for different angles of inclination and for an average irradiance of $630 \mathrm{~W} \cdot \mathrm{m}^{-2}$ and $B=15 \mathrm{mT}$.

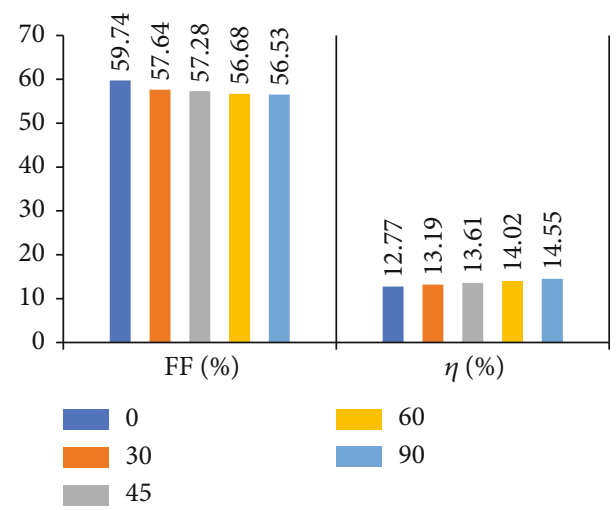

Figure 7: Fill factor and efficiency of the PV module for different angles of inclination $\alpha$ and for an average solar irradiance of $630 \mathrm{~W} \cdot \mathrm{m}^{-2}$ and $B=15 \mathrm{mT}$.

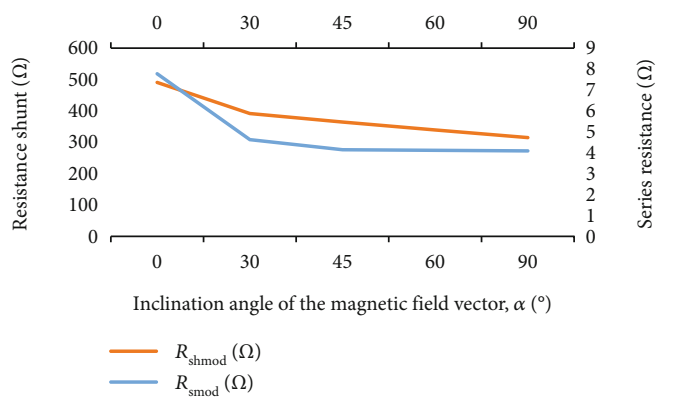

FIGURE 8: Series and shunt resistances against the inclination angle $\alpha$ of the MF vector and for an average irradiance of $630 \mathrm{~W} \cdot \mathrm{m}^{-2}$ and $B=15 \mathrm{mT}$.

\section{Conclusions}

Previous studies have established that the performance of silicon PV modules generally drops when mounted close to magnetic fields. Those studies, however, assumed the magnetic field to be aligned with the photosensitive area of the PV module; they did not take into account the effect of the direction of the MF vector on the performance of the PV module. The present experimental work was an attempt to fill that gap.

The current-voltage and power-voltage characteristic curves of a silicon PV module were drawn as a function of the inclination of the MF vector. Furthermore, the output of the PV module in terms of power produced, the fill factor, and the efficiency, among others, were calculated at various angles of inclination of the MF.

The experiment was conducted with a magnetic field of $15 \mathrm{mT}$, a mean solar irradiance of $630 \mathrm{~W} \cdot \mathrm{m}^{-2}$, and an average ambient temperature of about $39^{\circ} \mathrm{C}$. The angle of inclination $\alpha$ of the MF vector was varied with respect to the photosensitive face of the PV module.

The analysis of the results shows that as the angle of inclination $\alpha$ of the MF vector increases from 0 to $90^{\circ}$, the intensity of the currents $\left(I_{\max }\right.$ and $\left.I_{\mathrm{sc}}\right)$ increases while the voltages $\left(V_{\max }\right.$ and $\left.V_{\mathrm{oc}}\right)$ undergo a slight decrease. The resulting net effect is a decrease of the fill factor, the series and shunt resistances, and an increase of the power output and correspondingly the efficiency of the PV module.

As an illustration, when $\alpha$ moves from 0 to $90^{\circ}$, the relative decrease of the series and shunt resistances is $47.44 \%$ and $36 \%$, respectively, and that of the fill factor is $5.4 \%$. Within the same range of deviation of the angle of inclination of the MF vector, the power output and consequently the efficiency of the PV module increase of $14 \%$.

As the angles formed by the magnetic field and the photosensitive face of the module increases from 0 to $90^{\circ}$, the values of all the parameters are converging towards those recorded when there is no magnetic field. At $90^{\circ}$, they assume almost the same values as in the absence of the MF. These results indicate that when $\vec{B}$ is perpendicular to the junction of each cell of the PV module, its effect on the PV module vanishes.

These results are explained by the fact that it is the component $B_{y}$ of the MF vector which is parallel to the junction of each cell that acts on the minority carriers (electrons) thus negatively affecting the performance of the PV module. As $B_{y}$ decreases with increasing angle of inclination $\alpha$, the deviation of the electrons towards the lateral surfaces decreases, resulting in a decrease of the accumulation of electrons near the junction. Overall, the net influence of $\vec{B}$ on the operation of the PV module decreases with the increase of the inclination angle $\alpha$ of its vector.

It appears from this study that the effect of magnetic field on the performance of silicon PV module largely depends on the angle of inclination of its vector. It is therefore essential to take into account that angle when PV modules are to be installed in an environment where a magnetic field is present. In order to mitigate or cancel the effect of the MF on a silicon PV module, it is necessary that the magnetic field vector aligns towards a direction that is perpendicular to the photosensitive area of the module.

\section{Data Availability}

The data used to support the findings of this study are available from the corresponding author upon request. 


\section{Conflicts of Interest}

The authors declare that there is no conflict of interest regarding the publication of this paper.

\section{Acknowledgments}

The authors are grateful to BUF 01 of International Science Program (ISP) for supporting their research group (energy and environment).

\section{References}

[1] J. H. Wolgemuth and S. Kurtz, "Reliability testing beyond qualification as a key component in photovoltaic's progress toward grid parity," in Proceeding of the IEEE International Reliability Physics Symposium, Montetrey, California, 2011.

[2] M. Belarbi, A. Boudghene-Stambouli, E. Belarbi, and K. Haddouche, "A new algorithm of parameter estimation of a photovoltaic solar panel," Turkish Journal of Electrical Engineering and Computer Sciences, vol. 24, pp. 276-284, 2016.

[3] F. Dia, N. Mbengue, M. Diagne, O. A. Niasse, B. Ba, and C. Sene, "Contribution to the study of the degradation of modules PV in the tropical latitudes: case of Senegal," Research Journal of Applied Sciences, Engineering and Technology, vol. 12, no. 4, pp. 427-438, 2016.

[4] M. Zoungrana, I. Zerbo, B. Soro, M. Sawadogo, S. Tiendrebeogo, and D. J. Bathiebo, "The effect of magnetic field on the efficiency of a silicon solar cell under an intense light concentration," Advances in Science and Technology Research Journal, vol. 11, no. 2, pp. 133-138, 2017.

[5] S. E. Boukebbous and D. Kerdoun, "Study, modeling and simulation of photovoltaic panels under uniform and nonuniform illumination conditions," Revue des Energies Renouvelables, vol. 18, no. 2, pp. 257-268, 2015.

[6] I. Zerbo, M. Zoungrana, I. Sourabié, A. Ouédraogo, B. Zouma, and D. J. Bathiébo, "External magnetic field effect on bifacial silicon solar cell's electric power and conversion efficiency," Turkish Journal of Physics, vol. 39, no. 3, pp. 288-294, 2015.

[7] S. Madougou, F. Made, M. S. Boukary, and G. Sissoko, "I-V characteristics for bifacial silicon solar cell studied under a magnetic field," Advanced Materials Research, vol. 18-19, pp. 312-333, 2007.

[8] D. U. Combari, I. Zerbo, M. Zoungrana, E. W. Ramde, and D. J. Bathiebo, "Modelling study of magnetic field effect on the performance of a silicon photovoltaic module," Energy and Power Engineering, vol. 9, no. 8, pp. 419-429, 2017.

[9] Y. Betser, D. Ritter, G. Bahir, S. Cohen, and J. Sperling, "Measurement of the minority carrier mobility in the base of heterojunction bipolar transistors using a magnetotransport method," Applied Physics Letters, vol. 67, no. 13, pp. 1883-1884, 1995.

[10] S. Erel, "Comparing the behaviours of some typical solar cells under external effect," Teknoloji, vol. 11, no. 3, pp. 233-237, 2008.

[11] S. Erel, "The effect of electric and magnetic fields on the operation of a photovoltaic cell," Solar Energy Materials and Solar Cells, vol. 71, no. 2, pp. 273-280, 2002.

[12] R. R. Vardanyan, U. Kerst, P. Wawer, and H. Wagemann, "Method for measurement of all recombination parameters in the base region of solar cells," in Proceeding of 2nd World Conference and Exhibition on Photovoltaic Solar Energy Conversion, vol. 1, pp. 191-193., Vienna, Austria, July 1998.
[13] D. U. Combari, E. W. Ramde, I. Sourabie, M. Zoungrana, I. Zerbo, and D. J. Bathiebo, "Performance investigation of a silicon photovoltaic module under the influence of a magnetic field," Advances in Condensed Matter Physics, vol. 2018, Article ID 6096901, 8 pages, 2018.

[14] H. Fathabadi, "Impact of high-voltage power transmission lines on photovoltaic power production," Solar Energy, vol. 163, pp. 78-83, 2018.

[15] H. Fathabadi, "Effect of external AC electric and magnetic fields on the power production of a silicon solar cell," IEEE Journal of Photovoltaics, vol. 8, no. 6, pp. 1408-1412, 2018.

[16] H. Fathabadi, "Comparative study on the effect of magnetic field on the photocurrent density of organic, dye-sensitized and silicon solar cells," Journal of Materials Science: Materials in Electronics, vol. 30, no. 18, pp. 17314-17321, 2019.

[17] I. Sourabie, I. Zerbo, M. Zoungrana, D. U. Combari, and D. J. Bathiebo, "Effect of incidence angle of magnetic field on the performance of a polycrystalline silicon solar cell under multispectral illumination," Smart Grid and Renewable Energy, vol. 8, no. 10, pp. 325-335, 2017.

[18] M. S. Abdul Kareem and M. Saravanan, "A new method for accurate estimation of PV module parameters and extraction of maximum power point under varying environmental conditions," Turkish Journal of Electrical Engineering and Computer Sciences, vol. 24, pp. 2028-2041, 2016. 